\title{
Esgotamento físico dos profissionais de enfermagem no combate da COVID-19
}

RESUMO | Objetivo: Averiguar os fatores associados ao esgotamento físico dos profissionais de enfermagem no combate da COVID-19. Método: Trata-se de uma revisão integrativa. Realizou-se a busca por artigos; com delimitação nos últimos 5 anos; nos idiomas português, inglês e espanhol; disponíveis na íntegra. Nas seguintes plataformas de dados: BDENF; LILACS; MEDLINE/ BVS; PUBMED; Science Direct e na SciELO. Resultados: Os dados foram organizados e apresentados em figuras e tabelas. Dos 76 estudos encontrados, 2 estava disponível na BDENF; 2 na LILACS; 3 na MEDLINE/BVS; 5 na PUBMED; 61 na Science Direct e 3 na SciELO; contudo, após a leitura permaneceram apenas os que atendiam aos critérios para inclusão e exclusão descritos na metodologia, 8 estudos. Conclusão: A revisão elaborada proporcionou a identificação dos fatores associados ao esgotamento físico e psicológico dos profissionais de enfermagem ao combate da COVID-19; e frequentemente relacionados a sintomas de depressão, ansiedade, insônia e angústia.

Palavras-chaves: Esgotamento Profissional; Profissionais de Enfermagem; Infecções por Coronavirus.

\begin{abstract}
Objective: To investigate the factors associated with the physical exhaustion of nursing professionals in the fight against COVID-19. Method: This is an integrative review. The search for articles was carried out; with delimitation in the last 5 years; in Portuguese, English and Spanish; available in full. On the following data platforms: BDENF; LILACS; MEDLINENHL; PUBMED; Science Direct and SciELO. Results: The data were organized and presented in figures and tables. Of the 76 studies found, 2 were available from BDENF; 2 at LILACS; 3 at MEDLINENHL; 5 at PUBMED; 61 at Science Direct and 3 at SciELO; however, after reading, only those that met the inclusion and exclusion criteria described in the methodology remained, 8 studies. Conclusion: The elaborated review provided the identification of factors associated with the physical and psychological exhaustion of nursing professionals to combat COVID-19; and often related to symptoms of depression, anxiety, insomnia and distress.
\end{abstract}

Keywords: Burnout, Professional; Nurse practitioners; Coronavirus Infections.

RESUMEN | Objetivo: Investigar los factores asociados al agotamiento físico de los profesionales de enfermería en la lucha contra el COVID-19. Método: Esta es una revisión integradora. Se realizó la búsqueda de artículos; con delimitación en los últimos 5 años; en portugués, inglés y español; disponible en su totalidad. En las siguientes plataformas de datos: BDENF; LILACS; MEDLINENHL; PUBMED; Science Direct y SciELO. Resultados: Los datos fueron organizados y presentados en figuras y tablas. De los 76 estudios encontrados, 2 estaban disponibles en BDENF; 2 en LILACS; 3 en MEDLINENHL; 5 en PUBMED; 61 en Science Direct y 3 en SciELO; sin embargo, después de la lectura, solo quedaron aquellos que cumplieron con los criterios de inclusión y exclusión descritos en la metodología, 8 estudios. Conclusión: La revisión elaborada proporcionó la identificación de factores asociados al agotamiento físico y psicológico de los profesionales de enfermería para combatir el COVID-19; ya menudo se relaciona con síntomas de depresión, ansiedad, insomnio y angustia.

Palabras claves: Agotamiento Profesional; Profesionales de Enfermería; Infecciones por Coronavirus.

\section{Fabiana Maria Sena Santos}

Enfermeira (egressa) Faculdade da Escada (FAESC). Escada, Pernambuco (PE), Brasil. ORCID: 0000-0002-7384-9852

\section{Jessica Dantas Pessoa}

Discente do Curso de Bacharel em Enfermagem pelo Centro Universitário Maurício de Nassau (UNINASSAU). Recife, Pernambuco (PE), Brasil.

ORCID: 0000-0001-8376-7992

\section{Liniker Scolfild Rodrigues da Silva \\ Sanitarista na modalidade residência pela Faculdade de Ciências Médicas (FCM)/ Universidade de Pernambuco (UPE). Espe- cialista em Enfermagem Obstetra na moda- lidade residência pela Faculdade de Enfer- magem Nossa Senhora das Graças (FENSG)/ UPE. Recife, Pernambuco (PE), Brasil. ORCID: 0000-0003-3710-851X}

Maria Luciane Torres Honorio

Enfermeira (egressa) Faculdade da Escada (FAESC). Escada, Pernambuco (PE), Brasil. ORCID: 0000-0003-2110-0361

\section{Mariana Santos de Melo}

Discente do Curso de Bacharel em Enfermagem pela Faculdade da Escada (FAESC). Escada, Pernambuco (PE), Brasil.

ORCID: 0000-0001-5566-4160

\section{Nayane Alves do Nascimento}

Discente do Curso de Bacharel em Enfermagem pelo Centro Universitário Maurício de Nassau (UNINASSAU). Recife, Pernambuco (PE), Brasil.

ORCID: 0000-0001-6989-4629

Recebido em: 29/04/2021

Aprovado em: 14/05/2021
INTRODUÇÃO

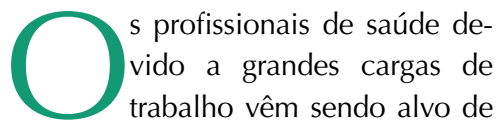
estudos, os enfermeiros vivem um grande estresse ocupacional, reduzindo a qualidade de vida, com a pandemia do COVID-19, o esgotamento profissional é uma grande preocupação para a saúde pública, pois é necessário equipes de saúde para atender as demandas já que houve uma explosão de casos da doença ${ }^{1}$.

Simultaneamente à pandemia, a Organização Mundial da Saúde (OMS) declarou o ano de 2020 como, o "Ano da Enfermagem", propondo uma campanha mundial chamada Nursing Now, em parceira com o Conselho Internacional de 
Enfermagem (ICN) e os órgãos de classe de diversos países. Tinha como objetivo enaltecer o papel da Enfermagem para atingir as metas de saúde pactuadas pelos países membros da ONU, e ainda elevar o status da Enfermagem, tem em vista sua importância para concepção e implementação das políticas de saúde ${ }^{2}$. Mostrando ainda mais o valor do profissional de enfermagem em tempos de pandemia.

Os fatores desencadeantes da depressão entre os profissionais de enfermagem podem estar associados ao processo de trabalho, como o turno, o relacionamento entre profissional-paciente, profissional-família e profissional-profissional, a sobrecarga de trabalho, o desgaste, o suporte social, o conflito de interesses e as estratégias de enfrentamento desenvolvidas $^{3}$. Diante disso, o presente estudo tem como objetivo averiguar os fatores associados ao esgotamento físico dos profissionais de enfermagem no combate da COVID-19.

\section{MÉTODO}

Trata-se de uma revisão integrativa desenvolvida com as seguintes etapas: (1) elaboração da questão norteadora e objetivo do estudo; (2) definição de critérios de inclusão e exclusão das produções científicas; (3) busca de estudos científicos nas bases de dados e bibliotecas virtuais; (4) análise e categorização das produções encontradas; (5) resultados e discussão dos achados ${ }^{4}$.

Para o levantamento da questão norteadora, utilizou-se a estratégia PICo, uma metodologia que auxilia na construção de uma pergunta de pesquisa e busca de evidências para uma pesquisa não-clínica, onde P = População / Paciente; I = Interesse; e $\mathrm{Co}=$ Contexto ( $\mathrm{P}$ : Profissionais de Enfermagem; I: Esgotamento físico no combate do COVID-19; Co: Melhores condições de trabalho). Desta forma, definiu-se a seguinte questão norteadora da pesquisa: "Quais os fatores relacionados ao esgotamento físico dos(as) enfermeiros(as) ao combate do COVID-19?".
Os fatores desencadeantes da depressão entre os profissionais de enfermagem podem estar associados ao processo de trabalho, como o turno, o relacionamento entre profissionalpaciente, profissional-família e profissionalprofissional, a sobrecarga de trabalho, o desgaste, o suporte social, o conflito de interesses e as estratégias de enfrentamento desenvolvidas.
Para seleção dos artigos, utilizaram-se como critérios de inclusão: artigo original, de abordagem qualitativa, disponíveis na íntegra, publicado nos últimos 5 anos em português, inglês ou espanhol, que respondessem ao objetivo do estudo. Foram excluídas as literaturas cinzas, bem como publicações repetidas de estudos em mais de uma base de dados e os artigos que não responderam à questão norteadora do estudo. Justifica-se a delimitação temporal nos últimos 5 anos por causa do período da pandemia da COVID-19, ou seja, pouco mais de 1 ano.

O levantamento dos dados ocorreu durante o mês de março e abril de 2021 nas seguintes Bases de Dados: Base de dados de Enfermagem (BDENF); Literatura Latino-Americana e do Caribe em Ciências da Saúde (LILACS); Medical Literature Analysis and Retrieval System Online via Biblioteca Virtual de Saúde (MEDLINE/BVS), PubMed, Science Direct e na Biblioteca virtual Scientific Electronic Library Online (SciELO).

Buscaram-se os artigos indexados a partir dos Descritores em Ciências da Saúde (DeCS): "Esgotamento Profissional", "Profissionais de Enfermagem", "Infecções por Coronavirus". Utilizaram-se os respectivos termos provenientes do Medical Subject Headings (MeSH): "Burnout, Professional", "Nurse Practitioners", "Coronavirus Infections". A operacionalização e a estratégia de busca se deram a partir da combinação com operador booleano AND e OR, efetuando a busca conjunta e individualmente para que possíveis diferenças fossem corrigidas (Quadro 1).

A seleção dos estudos baseou-se no Preferred Reporting Items for Systematic Review and Meta-Analyse (PRISMA)5, com o objetivo de auxiliar no desenvolvimento de artigos. A princípio eliminaram-se por meio da leitura de títulos e resumos, estudos duplicados. Destes pré-selecionados, realizou-se leitura na íntegra, a fim de verificar os que atendem à questão norteadora e aos critérios 
de inclusão/exclusão. Construiu-se então a amostra final com estudos pertinentes aos critérios pré-estabelecidos (Figura 1).

Após a leitura dos artigos selecionados os estudos foram categorizados, classificando o conhecimento produzido em níveis de evidência de acordo com Melnyk BM e Fineout-Overholt E6: nível I, as evidências são relacionadas à revisão sistemática ou metanálise de ensaios clínicos randomizados controlados ou provenientes de diretrizes clínicas baseadas em revisões sistemáticas de ensaios clínicos randomizados controlados; no nível II, evidências derivadas de no mínimo um ensaio clínico randomizado controlado bem delineado; no nível III, evidências de ensaios clínicos bem delineados sem randomização; no nível IV, evidências advindas de estudos de coorte e de caso-controle bem delineados; no nível V, evidências provenientes de revisão sistemática de estudos descritivos e qualitativos; no nível VI, evidências derivadas de um único estudo descritivo ou qualitativo; e no nível VII, evidências derivadas de opinião de autoridades e/ou relatório de comitês de especialistas.

Obteve-se a sumarização das informações do corpus por meio de um instrumento: identificação do artigo original; autoria do artigo; ano de publicação; país; características metodológicas do estudo; e amostra do estudo. Analisaram-se os da- dos com o software Interface de $\mathrm{R}$ pour les Analyses Multidimensionnelles de Textes et de Questionnaires (IRAMUTEQ), versão 7.0, que possibilitou a análise da Classificação Hierárquica Descendente (CHD).

Objetivando uma melhor compreensão e visualização dos principais achados organizaram-se os dados apresentando-os em figuras e tabelas, expostos de forma descritiva.

\section{RESULTADOS}

Os estudos levantados estão dispostos evidenciando seus títulos, autores, anos de publicação, níveis de evidências, objetivos e resultados. Após a leitura dos

Quadro 1 - Estratégia de busca por base de dados. Recife, Pernambuco (PE), Brasil, 2021.

\begin{tabular}{|c|c|c|c|}
\hline Bases de dados & Termos da busca & Resultados & Selecionados \\
\hline BDENF & Esgotamento Profissional AND Profissionais de Enfermagem AND Infecções por Coronavirus & 2 & 1 \\
\hline LILACS & Esgotamento Profissional AND Profissionais de Enfermagem AND Infecções por Coronavirus & 2 & 0 \\
\hline MEDLINE/BVS & Esgotamento Profissional AND Profissionais de Enfermagem AND Infecções por Coronavirus & 3 & 1 \\
\hline Science Direct & Burnout, Professional AND Nurse practitioners AND Coronavirus Infections & 61 \\
\hline PubMed & Burnout, Professional AND Nurse practitioners AND Coronavirus Infections & 5 & 1 \\
\hline SciELO & Burnout, Professional OR Nurse practitioners AND Coronavirus Infections & 3 \\
\hline Total & & 8 & 76 \\
\hline Fonte: Dados da pesquisa, 2021. & \\
\hline
\end{tabular}

Figura 1 - Fluxograma do processo de seleção dos estudos primários adaptado do PRISMA. Recife, Pernambuco (PE), Brasil, 2021.

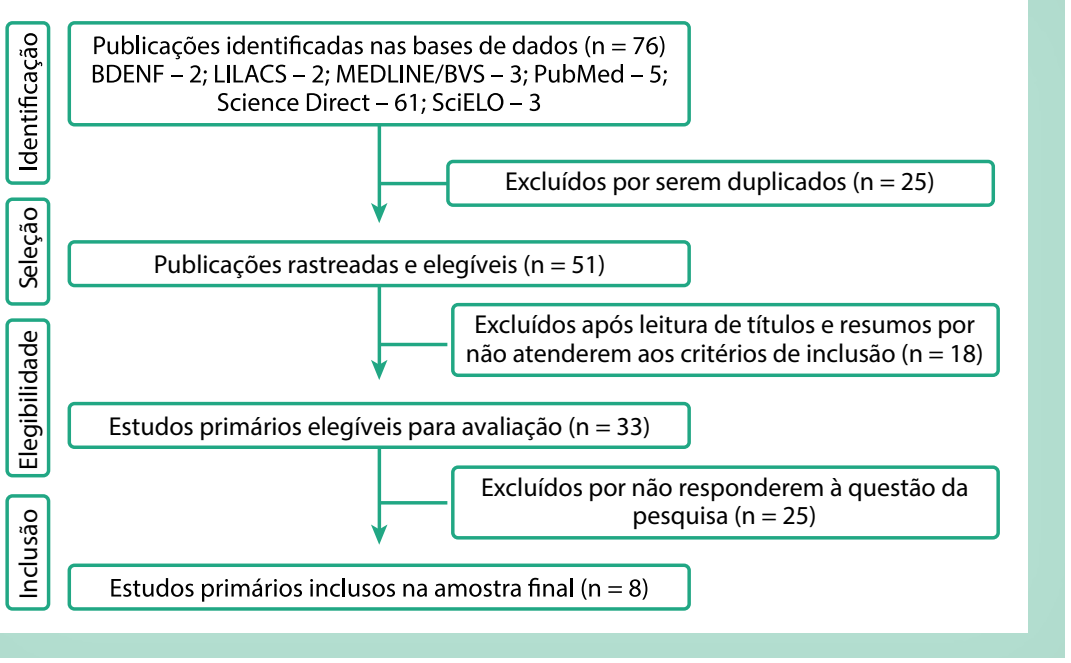

Fonte: Dados da pesquisa, 2021. artigos selecionados, os estudos foram categorizados, classificando o conhecimento produzido sobre o tema, em níveis de evidência, majoritariamente nível IV - evidências advindas de estudos de coorte e de caso-controle bem delineados. Os principais achados dispostos nos objetivos e conclusões, estão diretamente associados aos profissionais de enfermagem que sofrem grandemente com esgotamento físico e psicológico consequente da sobrecarga de trabalho ao combate do COVID-19 (Tabela 1).

\section{DISCUSSÃO}

Foi possível observar alguns fatores associados ao esgotamento físico e psicológico dos profissionais de enfermagem nos artigos que fizeram parte desde estu- 
Tabela 1 - Sintese dos principais achados sobre esgotamento físico dos profissionais de enfermagem ao combate do COVID-19. Recife, Pernambuco (PE), 2021.

N Título / Base

Burnout e seus fatores de influência entre enfermeiras da

1 linha de frente e enfermeiras de outras enfermarias durante 0 surto da Doença do Coronavírus -COVID-19- no Irã. / MEDLINE

\author{
Autores (Ano) \\ Nível de \\ evidência
}

Objetivo

Resultados
Sarboozi Hoseinabadi, Tahere et al., (2020) ${ }^{7}$
IV

Avaliar o nível de burnout durante um surto de COVID-19 e para identificar os fatores de influência entre enfermeiras da linha de frente e enfermeiras de outras enfermarias.
0 nível de burnout em enfermeiras de linha de frente era maior do que outras enfermeiras, o fator de influência mais importante foi o estresse no trabalho. Em relação aos efeitos negativos do burnout em enfermeiros de saúde física e mental, sugere-se que uma estratégia forte seja considerada para reduzir o burnout dos enfermeiros para ser capaz de controlar surtos em andamento e futuros com sucesso.
Dimensões laborais, éticas e

2 políticas do dimensionamento de pessoal de enfermagem diante da COVID-19. / BDENF
Nishiyama, Juliana Aparecida Peixoto et al., $(2020)^{8}$

\section{I}

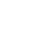

(n)

Propor discussão ampliada a respeito de dimensões que envolvem o dimensionamento de pessoal de enfermagem, articulando-as à realidade da pandemia por COVID-19.
Propor discussão ampliada a respeito de dimensões que envolvem o dimensionamento de pessoal de enfermagem, articulando-as à realidade da pandemia por COVID-19.
Desafios de profissionais de Goes, Fernanda

3 Enfermagem Pediátrica frente à Garcia Bezerra pandemia da COVID-19. / SciELO et al., $(2020)^{9}$
Identificar os desafios de profissionais de Enfermagem

Pediátrica frente à pandemia da COVID-19.
Distintos desafios referentes à pandemia da COVID-19 foram relatados, dentre eles, a promoção de uma assistência integral e de qualidade frente à preocupação quanto à proteção de si e do outro, com destaque para o sentimento de medo. A falta de equipamentos de proteção individual, treinamentos, testes diagnósticos e conhecimentos/informações relacionados à doença, o número reduzido de profissionais de Enfermagem e a desvalorização da categoria também foram sinalizados.
Enfermeiros líderes empregam práticas contemplativas para

4 promover o bem-estar do profissional de saúde e diminuir a ansiedade. / PubMed

\section{Cunningham \\ T, Çayir E., $(2021)^{10}$}

III
Testar a eficácia de um retiro de resiliência de um dia nos níveis de ansiedade dos profissionais de saúde, intenção de se envolver em práticas de atenção plena e autoeficácia em relação à atenção plena.
Retiros breves de resiliência endossados pela liderança de enfermagem podem reduzir a ansiedade percebida e facilitar o envolvimento em práticas contemplativas, que estão associadas a uma diminuição no risco de burnout.
Um estudo fenomenológico da

5 experiência vivida por enfermeiras na batalha do COVID-19. I Science Direct
Explorar a experiência vivida Gunawan, Joko IV por enfermeiras no combate et al., $(2021)^{11}$ ao COVID-19 em Belitung, Indonésia.
Os resultados forneceram perspectivas holísticas que descrevem os sentimentos da enfermeira e o estado atual dos fenômenos no ambiente hospitalar e comunitário. As emoções que os enfermeiros percebem são mescladas de sentimentos negativos a positivos com a mudança. 
Impacto da pandemia da doença coronavírus de 2019 na

6 depressão, ansiedade e níveis de estresse de trabalhadores de cuidados de saúde intensivos. I Science Direct

Determinar os níveis de depressão, ansiedade e sintomas de estresse e fatores associados à

Hammond,

Naomi E. et al., IV $(2020)^{12}$ carga psicológica entre profissionais de saúde de cuidados intensivos nos estágios iniciais da pandemia de doença coronavírus de 2019.
Efeitos psicológicos do surto de COVID-19 em enfermeiras que

7 trabalham em hospitais terciários femininos e infantis de Sichuan, China: um estudo transversal. I Science Direct
Examinar o estado psicológico de enfermeiras que trabalham em hospitais infantis e femininos terciários em Sichuan, China, a fim de explorar o possível efeito do COVID-19.

He, Qiuyang et
al., $(2021)^{13}$ IV

Entre 22 e $29 \%$ dos entrevistados relataram sintomas de depressão, ansiedade e estresse moderados a extremamente graves, com mulheres relatando pontuações mais altas do que homens. Embora o gênero feminino pareça desempenhar um papel, fatores modificáveis também contribuem para a carga psicológica e devem ser mais estudados.

Os resultados sugerem que a epidemia de COVID-19 não afeta necessariamente a saúde psicológica das enfermeiras que trabalham em hospitais infantis e femininos em Sichuan. Os resultados deste estudo podem servir como sugestões valiosas para direcionar a promoção do bem-estar psicológico entre os enfermeiros-alvo.

0 impacto psicossocial nas enfermeiras da linha de frente 8 ao cuidar de pacientes com Covid-19 durante a primeira onda da pandemia na cidade de Nova York. / Science Direct
Investigar os fatores pessoais

Kovner, Christine et al., IV $(2021)^{14}$ e contextuais associados ao funcionamento psicológico de enfermeiras atendendo a Covid na área da cidade de Nova York.
A compreensão dos potenciais gatilhos e fatores de vulnerabilidade pode informar o desenvolvimento de recursos institucionais que ajudem a minimizar o seu impacto, reduzindo o risco de morbilidade psicológica.

Fonte: Dados da pesquisa, 2021

do, um dos fatores mais importantes foi o estresse no ambiente de trabalho; falta de dimensionamento da equipe; a falta de equipamentos de proteção individual, treinamentos, testes diagnósticos e conhecimentos/informações relacionados à doença, o número reduzido de profissionais de Enfermagem e a desvalorização da categoria.

Um estudo apontou inúmeros desafios relacionados à pandemia da $\mathrm{CO}$ VID-19 percebidos pelos profissionais de Enfermagem que prestam assistência pediátrica, destacando-se a promoção de uma assistência integral e de qualidade diante da preocupação com a proteção de si e do outro, se faz presente o sentimento de medo ${ }^{9}$. Corroborando, outro estudo constatou que os profissionais de enfermagem com ansiedade, em sua maioria, são técnicos em cargo assistencial $(44,2 \%)$, atuam em setores críticos $(55,8 \%)$, são concursados $(44,2 \%)$ e trabalham na área da saúde há mais de 10 anos $(27,9 \%)$. Com isso, que setores de atuação profissional e situações do ambiente podem provocar ansiedade, tendo
66

os profissionais de enfermagem com ansiedade, em sua maioria, são técnicos em cargo assistencial $(44,2 \%)$, atuam em setores críticos (55,8\%), são concursados $(44,2 \%)$ e trabalham na área da saúde há mais de 10 anos $(27,9 \%)$ destaque os setores fechados e com procedimentos de alta complexidade, bem como a instabilidade ou agravamento do estado de saúde dos pacientes ${ }^{3}$.

Ainda, há relatos dos profissionais da saúde sobre as más condições de trabaIho, jornadas exaustivas, falta de treinamentos para novos protocolos, carência de materiais de proteção tornando, assim, um ambiente de trabalho hostil e com isto afetando diretamente a saúde da equipe no combate ao Covid-19 ${ }^{15}$.

Um estudo transversal, com 1.257 profissionais de saúde em 34 hospitais equipados com clínicas ou enfermarias para pacientes com COVID-19 em várias regiões da China, encontrou uma proporção considerável de profissionais de saúde com sintomas de depressão, ansiedade, insônia e angústia. Entre o grupo que mais sofria estavam as mulheres, enfermeiras, pessoas que moravam em Wuhan e profissionais de saúde envolvidos no diagnóstico, no tratamento ou na prestação de cuidados de enfermagem a pacientes com suspeita ou confirmação de COVID-19 ${ }^{16}$. Assim, a preocupação 
com esse aspecto de sanidade da população demandou de alguns países a oferta de assistência especializada em saúde mental ${ }^{17}$.

A análise da situação da força de trabalho em saúde no Brasil tem sido feita em vários estudos, que apontam os principais problemas, tanto o que diz respeito à disponibilidade e distribuição das diversas categorias profissionais para atender às necessidades de funcionamento adequado dos serviços, nos diversos níveis de atenção, quanto os problemas relacionados à gestão do trabalho, isto é, os mecanismos de contratação, qualificação e valorização da força de trabalho no setor ${ }^{18}$.
Uma limitação para o estudo foi o tamanho da amostra e a disponibilidade de artigos científicos para comparação dos resultados. Fazendo-se necessário a realização de mais estudos contendo uma amostra maior e possibilitando discussão acerca das implicações relacionadas aos impactos da COVID-19 na saúde dos profissionais de Enfermagem que atuam na linha de frente.

\section{CONCLUSÃO}

A revisão elaborada proporcionou a identificação dos fatores associados ao esgotamento físico e psicológico dos profissionais de Enfermagem ao combate da COVID-19; e frequentemente relacionados a sintomas de depressão, ansiedade, insônia e angústia. No entanto, há escassez de estudos que dão a verdadeira importância a saúde desses profissionais, essenciais na prevenção, promoção da saúde, e educação permanente, ainda que esse número tenha aumentado progressivamente nos últimos anos. Portanto, este estudo proporcionou a percepção de que ainda que tímido, há um crescimento no número de estudos que valorizam a saúde dos profissionais em questão de forma integral.

\section{Referências}

1. Brasil. Ministério da Saúde. Boletim Epidemiológico 11 - COE-COVID19-17 de abril de 2020. [Internet]. 2020. Available from: https://www.saude.gov.br/ images/pdf/2020/April/18/2020-04-17---BE11---Boletim-doCOE-21h.pdf 2. World Health Organization (WHO). [Homepage]. World Health Organization; 2020 [cited 2021 apr 22]. Available from: https://www.who.int/

3. Dal'Bosco EB, Floriano LSM, Skupien SV, Arcaro G, Martins AR, Anselmo ACC Correa. A saúde mental da enfermagem no enfrentamento da COVID-19 em um hospital universitário regional. Rev. Bras. Enferm. [Internet]. 2020 [cited 2021 Apr 22]; 73(Suppl 2): e20200434. Available from: https://doi. org/10.1590/0034-7167-2020-0434.

4. Lisboa MT. Elements to formulate a research design. Mural Internacional Rio de Janeiro, 2019; 10:e38439. https://doi.org/10.12957/rmi.2019.38439

5. Barbosa FT, Lira AB, Oliveira Neto OB, Santos LL, Santos IO, Barbosa LT et al. Tutorial for performing systematic review and meta-analysis with interventional anesthesia studies. Brazilian Journal of Anesthesiology (English Edition), 2019; 69(3): 299-306. https://doi.org/10.1016/j.bjan.2018.11.007

6. Melnyk BM, Fineout-Overholt E. Making the case for evidence-based practice. In B. M. Melnyk \& E. Fineout-Overholt. Evidence-based practice in nursing \& healthcare: a guide to best practice. 2005; 3-24. Philadelphia: Lippincot Williams \& Wilkins.

7. Sarboozi Hoseinabadi T, Kakhki S, Teimori G, NayyeriS. Burnout and its influencing factors between frontline nurses and nurses from other wards during the outbreak of Coronavirus Disease -COVID-19- in Iran. invest. educ. enferm [Internet]. 2020Jul.10 [cited 2021Apr.21];38(2). Available from: https://revistas.udea.edu.co/index.php/iee/article/view/342790

8. Nishiyama JAP, Moraes RMR, Magalhães AMM, Nicola AL, Trevilato DD, Oliveira JL. Dimensões laborais, éticas e políticas do dimensionamento de pessoal de enfermagem diante da COVID-19. Esc. Anna Nery [Internet]. 2020 [cited 2021 Apr 21]; 24(spe): e20200382. Available from: http://dx.doi.org/10.1590/ 2177-9465-ean-2020-0382.

9. Góes FGB, Silva ACSS, Santos AST, Pereira-Ávila FMV, Silva LJ, Silva LF et al. Challenges faced by pediatric nursing workers in the face of the COVID-19 pandemic. Rev. Latino-Am. Enfermagem [Internet]. 2020 [cited 2021 Apr 21] 28: e3367. Available from: $h t t p: / / d x . d o i . o r g / 10.1590 / 1518-8345.4550 .3367$.

10. Cunningham T, Çayir E. Nurse Leaders Employ Contemplative Practices to Promote Healthcare Professional Well-being and Decrease Anxiety. J Nurs Adm. [Internet]. 2021 Mar 1 [cited 2021 Apr 21]; 51(3):156-161. Available from: http://dx.doi.org/10.1097/NNA.0000000000000987. PMID: 33570373.

11. Gunawan J, Aungsuroch $Y$, Marzilli C, Fisher ML, Nazliansyah, Sukarna A. A phenomenological study of the lived experience of nurses in the battle of COVID-19. Nurs Outlook. [Internet]. 2021 Feb 5 [cited 2021 Apr 21]; S0029-6554(21)00024-5. Available from: http://dx.doi.org/10.1016/j. outlook.2021.01.020.

12. Hammond NE, Crowe L, Abbenbroek B, Elliott R, Tian DH, Donaldson LH, et al. Impact of the coronavirus disease 2019 pandemic on critical care healthcare workers' depression, anxiety, and stress levels. Australian Critical Care [Internet]. 2021 [cited 2021 Apr 21]; 34(2):146-154. Available from: http://dx.doi. org/10.1016/j.aucc.2020.12.004.

13. He Q, Ren J, Wang G, Zhang J, Xiang J, He D. Psychological effects of the COVID-19 outbreak on nurses working in tertiary women's and children's hospitals from Sichuan, China: a cross-sectional study. International Journal Of Disaster Risk Reduction, [Internet]. 2021 [cited 2021 Apr 21]; 58 :102188. Available from: http://dx.doi.org/10.1016/j.ijdrr.2021.102188.

14. Kovner C, Raveis VH, Van Devanter N, Yu G, Glassman K, Ridge LJ. The psychosocial impact on frontline nurses of caring for patients with Covid-19 during the first wave of the pandemic in New York City. Nursing Outlook, [S.L.], v. 1, n. 1, p. 1-1, abr. 2021. Elsevier BV. http://dx.doi.org/10.1016/j. outlook.2021.03.019.

15. Filho JMJ, Assunção AA, Algranti E, Saito CA, Maeno M. A saúde do trabalhador e o enfrentamento da COVID-19. Revista Brasileira de revisão integrativa revista enfermagem atual in derme | edição especial COVID19 - 2020 e-020012 Saúde Ocupacional [Internet]. 2020 [acesso em 18 mai 2020];45, 1-3. Available from: https://www.scielo.br/scielo.php?script =sci_arttext\&pi$d=$ S0303- 76572020000100100 .

16. Lai J, Ma S, Wang Y, Cai Z, Hu J, Wei N et al. Factors Associated With Mental Health Outcomes Among Health Care Workers Exposed to Coronavirus Disease 2019. JAMA 2020; 3(3):e203976.

17. Martins Torres RA, da Conceição Bezerra Brito Veras K, Darc Martins Torres J, Dias Pimentel Gomes E, Vasconcelos Martins J, Carvalho Soeiro AC. Saúde mental das juventudes e COVID-19: discursos produtores do webcuidado educativo mediados na webrádio. Nursing [Internet]. $25^{\circ}$ de novembro de 2020 [citado 27 de abril de 2021];23(270):4887-96. Disponível em: http://revistas. mpmcomunicacao.com.br/index.php/revistanursing/article/view/1025 18. Teixeira Carmen Fontes de Souza, Soares Catharina Matos, Souza Ednir Assis, Lisboa Erick Soares, Pinto Isabela Cardoso de Matos, Andrade Laíse Rezende de et al. A saúde dos profissionais de saúde no enfrentamento da pandemia de Covid-19. Ciênc. saúde coletiva [Internet]. 2020 Sep [cited 2021 Apr 22]: 25(9): 3465-3474. Available from: https://doi.org/10.1590/141381232020259.19562020. 